Background Compared to UK white European adults, UK black African-Caribbean adults have higher mean systolic (SBP) and diastolic (DBP) blood pressure; UK South Asian adults have higher mean DBP but lower SBP. However, information on blood pressure in UK children from different ethnic groups is limited. The aim of this study was to compare blood pressure levels in UK children of black African-Caribbean, South Asian and white European origin.

Methods A cross sectional study of 5,666 UK primary school children of South Asian, black African Caribbean, and white European origin aged 9 to 10 years was undertaken. Ethnic and socioeconomic differences in SDP and DBP (as means and differences with their $95 \%$ confidence intervals) were obtained from multilevel linear regression models fitting school as a random effect in order to take account of the natural clustering of children within school. All analyses were adjusted for sex, age, month of assessment, blood pressure observer, room temperature and time of day of measurement fitted as fixed effects. The effects of adjustment for height, adiposity (fat mass index, sum of skinfolds) fitted as continuous variables, and socioeconomic circumstances on ethnic differences in blood pressure were then explored.

Results After adjustment for height and adiposity, black AfricanCaribbean children had a lower mean SBP than white Europeans (mean difference $1.62 \mathrm{mmHg}, 95 \%$ CI $0.86,2.38 \mathrm{mmHg}$ ), while mean DBP was similar (mean difference $0.58 \mathrm{mmHg}, 95 \% \mathrm{CI}-0.12$, $1.28 \mathrm{mmHg}$ ). The mean SBP difference was particularly marked in black African children. In similar analyses, South Asian children had a lower mean SBP (mean difference $1.10 \mathrm{mmHg}$, 95\%CI 0.34, 1.86 $\mathrm{mmHg}$ ) than white Europeans and a higher mean DBP (mean difference $1.07 \mathrm{mmHg}, 95 \% \mathrm{CI} 0.37,1.76 \mathrm{mmHg}$ ). The mean DBP difference was particularly marked among Indian and Bangladeshi, rather than Pakistani, children. Blood pressure was largely unrelated to socioeconomic circumstances; the ethnic differences in blood pressure were not affected by socioeconomic adjustment.

Conclusion A blood pressure pattern similar to that in adults is present in UK South Asian but not in UK black African-Caribbean children at 9-10 years. This suggests that key determinants of ethnic differences in blood pressure operate at different stages of the life course in these different ethnic groups. Understanding the reasons for the early emergence of ethnic differences in blood pressure (particularly among South Asians) is an important research priority.

\section{OP30 ARE THE ASSOCIATIONS BETWEEN ADIPOSITY, INSULIN RESISTANCE AND CIRCULATING GLUCOSE CONCENTRATIONS IN CHILDHOOD INFLUENCED BY ETHNICITY? EVIDENCE FROM THE CHILD HEART AND HEALTH STUDY IN ENGLAND (CHASE)}

doi:10.1136/jech-2012-201753.030

${ }^{1} \mathrm{CM}$ Nightingale, ${ }^{1} \mathrm{AR}$ Rudnicka, ${ }^{1} \mathrm{CG}$ Owen, ${ }^{2} \mathrm{~N}$ Sattar, ${ }^{1} \mathrm{DG}$ Cook, ${ }^{1} \mathrm{PH}$ Whincup. ${ }^{1}$ Division of Population Health Sciences and Education, St George's, University of London, London, UK; ${ }^{2}$ British Heart Foundation Glasgow Cardiovascular Research Centre, University of Glasgow, Glasgow, UK

Background Ethnic differences in type 2 diabetes risk between South Asians and white Europeans originate before adult life. Although South Asian adults have greater metabolic sensitivity to adiposity this has been little studied in childhood. We have therefore examined the associations between adiposity, insulin and glycated haemoglobin $(\mathrm{HbA1c})$ levels in children of South Asian and white European origin. Data on children of black African-Caribbean origin are also reported.

Methods This was a cross-sectional school-based study of 4633 nine and ten year-old children (response rate 68\%) predominantly of South Asian, black African-Caribbean and white European origin. Participants had detailed assessments of adiposity (including body mass index, skinfold thicknesses and fat mass index from bioimpedance) and provided fasting blood samples for assessment of HbA1c and insulin resistance (Homeostatic model assessmentinsulin resistance; HOMA-IR). Associations between HOMA-IR (log transformed), HbA1c and adiposity markers were estimated using multilevel linear regression.

Results All adiposity measures were positively associated with insulin resistance in all ethnic groups. However there were clear ethnic differences in the strength of association ( $p$ ethnic difference $<0.001$ ). The percentage increase in HOMA-IR for a one SD increase in fat mass index was $36.3 \%$ (95\% CI $32.1 \%$, 40.5\%) for South Asians and 25.1\% (95\% CI 21.1\%, 29.3\%) for white Europeans; black African-Caribbeans were similar to white Europeans $(26.4 \%$, $95 \%$ CI $22.5 \%, 30.4 \%)$. All adiposity markers were positively associated with $\mathrm{HbA1c}$ in both South Asians and black African-Caribbeans: the percentage increase in $\mathrm{HbA1c}$ for a one SD increase in fat mass index in was $0.04 \%$ (95\% CI $0.02 \%, 0.06 \%)$ in both ethnic groups. However there was no association among white Europeans (0.01\%, 95\% CI 0.00\%, 0.03\%).

Conclusion Even in childhood, both insulin resistance and HbA1c levels appear more sensitive to adiposity in South Asians. Among children of black African-Caribbean origin, HbA1c levels but not insulin resistance appear more sensitive to adiposity. The reasons for these differences in sensitivity to adiposity need to be understood. The results imply that early prevention of childhood obesity among South Asians and black African-Caribbeans is a particular priority for future control of type 2 diabetes in these high risk ethnic groups.

\section{OP31 ETHNIC DIFFERENCES IN THE DEVELOPMENT OF DISABILITY OVER 20 YEARS: RESULTS FROM THE SABRE STUDY}

doi:10.1136/jech-2012-201753.03

ED Williams, T Tillin, N Chaturvedi. International Centre for Circulatory Health, Imperial College London, London, UK

Background As life expectancy increases, healthy ageing becomes more salient, and therefore it is important to understand how conditions such as disability may affect the later years of our extended lives. Ethnic differences in disability have been observed in some countries, however there is a lack of evidence from British ethnic groups.

Methods Follow-up data over 20 years from 1789 White, Indian Asian and African Caribbean men and women were examined from a community-based study in West London. Disability was measured using the performance-based test of locomotor function and selfreported functional limitation, instrumental and basic activities of daily living (IADL/ADL) questionnaires. Logistic regression analyses examined ethnic group differences in disability, adjusting for socioeconomic, behavioural, adiposity and chronic disease risk factors.

Results After full adjustment, Indian Asian people were significantly more likely to have developed all of the disability outcomes, compared with UK Whites (locomotor dysfunction: OR 2.20, 95\% CI 1.56-3.11; functional limitation: OR 2.77, 2.01-3.81; IADL impairment: OR 3.12, 2.20-4.41; ADL impairment: OR 1.56, 1.112.24). Health behaviours, central adiposity, and chronic disease burden explained only a proportion of this excess risk. There were no ethnic group differences in locomotor dysfunction, functional limitation and IADL impairment between African Caribbean and White participants, however African Caribbean people showed a reduced risk of $\mathrm{ADL}$ impairment (OR 0.59, 0.38-0.93), after multivariate adjustment.

Conclusion These findings demonstrate dramatic ethnic group differences in performance-based and self-reported disability between White, Indian Asian, and African Caribbean people in the UK. The excessive risk experienced by Indian Asian people was partly explained by health behaviours, adiposity, and chronic 